\title{
Cement-a-TAN Temporary Proximal Femoral Replacement for Staged Joint Replacement - A Case Report
}

\author{
Sean D. Scattergood ${ }^{1}$, James W. A. Fletcher ${ }^{1}$, Sanchit A. Mehendale ${ }^{1}$, Stephen R. Mitchell ${ }^{1}$
}

\section{Learning Point of the Article:}

The Cement-a-TAN is an excellent, unique temporary spacing technique in staged proximal femoral replacement for infection in the proximal femur associated with significant bone loss, and orthopedic surgeons should be aware of how to construct this implant as a treatment option in these difficult cases.

\section{Abstract}

Introduction: Infected non-unions of proximal femoral fractures are difficult to treat. If debridement and revision fixation is unsuccessful, staged revision arthroplasty may be required. Non-viable tissue must be resected; coupled with the introduction of an antibiotic-eluting temporary spacer before definitive reconstruction. Tissue microbiological diagnosis and targeted antibiotic therapy are required. In cases of significant proximal femoral bone loss, spacing options are limited.

Case Report: We present a case of a bisphosphonate-induced subtrochanteric fracture that progressed to infected non-union. Despite multiple washouts and two revision fixations, the infection remained active with an unfavorable antibiogram. The patient required staged revision arthroplasty including a proximal femoral resection. To improve function through maintaining leg length and offset, the Cement-a-TAN was fabricated. It is a custom-made antibiotic-eluting articulating temporary spacer. Using a trochanteric entry cephalocondylar nail as a scaffold, bone cement was molded around the nail to fashion an anatomical and patient-specific proximal femoral spacer. Following resolution of the infection, the Cement-a-TAN was removed and a proximal femoral arthroplasty was successfully performed.

Conclusion: Cement-a-TAN is an excellent temporary spacing technique in staged proximal femoral replacement for infected non-union of the proximal femur where there has been significant bone loss. It preserves mobility and maintains leg length, offset, and periarticular soft-tissue tension, while offering good stability.

Keywords: Proximal femoral replacement, infection, hip, nail, spacer.

\section{Introduction}

Infected non-union of proximal femoral fractures is difficult to treat: If debridement and revision fixation is unsuccessful then staged arthroplasty is indicated $[1,2,3,4,5]$. Stage one involves debridement of non-viable bone and soft tissue, multiple sampling for microbiology and insertion of an antibioticeluting temporary spacer $[3,5,6]$. This ensures preservation and appropriate tensioning of the soft-tissue envelope, targeted delivery of a high concentration of antibiotics to the local area, systemic antibiotic therapy, reduces dead space, and allows weight-bearing which prevents the complications associated with immobility and potential prolonged bed rest $[3,4,7,8]$.
Following resolution of infection, the second stage, proximal femoral replacement, may be performed $[3,4,6]$.

If bone loss is minimal, standard spacers and commercially available devices may be sufficient but in cases of extensive proximal femoral bone loss, options are limited: There are few temporary spacing options described in the literature for cases of severe proximal femoral bone loss in the context of infection $[3,4,6,7]$.

We present a case of an atypical, subtrochanteric fracture that progressed to an infected non-union. Two-stage revision arthroplasty with proximal femoral replacement was required for salvage. By use in the first stage of a custom-made, antibiotic-
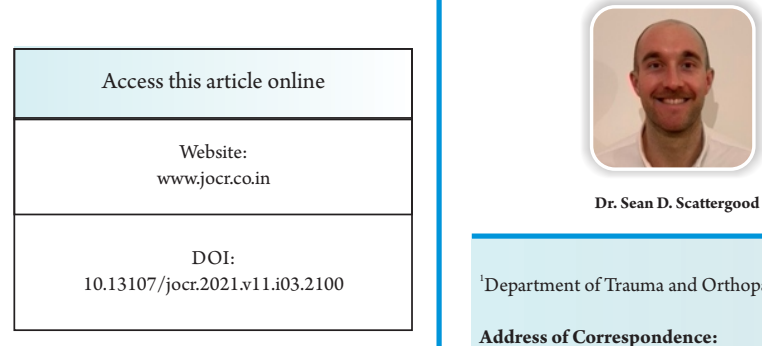

Dr. Sean D. Scattergood

Address of Correspondence:

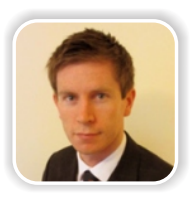

Dr. James W. A. Fletcher

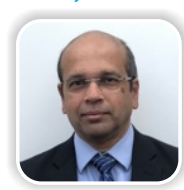

Dr. Sanchit A. Mehendale

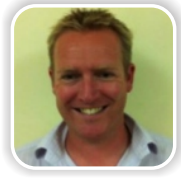

Dr. Stephen R. Mitchel

'Department of Trauma and Orthopaedics, Bristol Royal Infirmary, Bristol. UK.

Dr. Sean D. Scattergood,

Department of Trauma and Orthopaedics, Bristol Royal Infirmary, Bristol. UK.

E-mail: scattergood.sean@gmail.com

Journal of Orthopaedic Case Reports | pISSN 2250-0685 | eISSN 2321-3817 | Available on www.jocr.co.in | doi:10.13107/jocr.2021.v11.i03.2100 This is an Open Access article distributed under the terms of the Creative Commons Attribution Non-Commercial License (http://creativecommons.org/licenses/by-nc/3.0) which permits unrestricted non-commercial use, distribution, and reproduction in any medium, provided the original work is properly cited. 


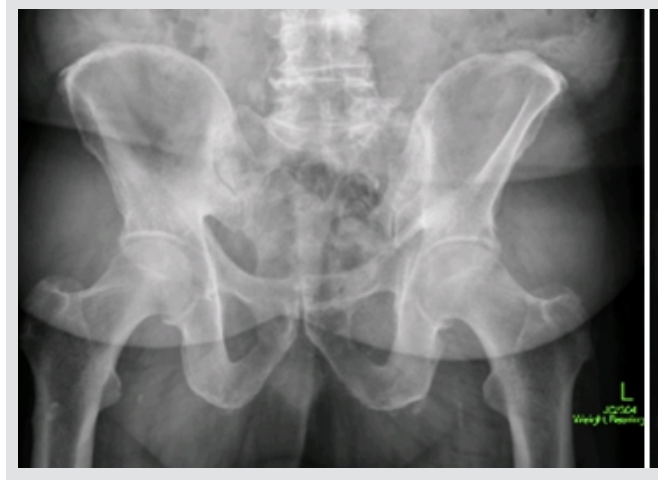

Figure 1: Coronal radiographs showing impending subtrochanteric fracture following a fall.

eluting, temporary spacer designed around a trochanteric antegrade nail, interstage morbidity was reduced while the infection was treated. Subsequently, a proximal femoral replacement type hip arthroplasty was successfully performed.

\section{Case Report}

We present the case of a 68-year-old male with a background of psoriatic arthritis for which he was medicated with regular leflunomide and intermittent courses of steroids for symptomatic flares. He had also taken alendronic acid for 10 years before it being discontinued 6 months before his fracture, as his dualenergy X-ray absorptiometry scan confirmed normal bone density.

Following a fall from standing height, the patient had initially been asymptomatic but gradually developed pain in the right hip on weight-bearing over the subsequent 2 weeks, leading to his delayed presentation. On assessment, he had pain over the lateral aspect of his right hip in the subtrochanteric region, with radiographs demonstrating an impending fracture (Fig. 1). At this stage, the patient was advised that a prophylactic nail was indicated but declined surgical intervention at that stage to travel abroad on business matters.

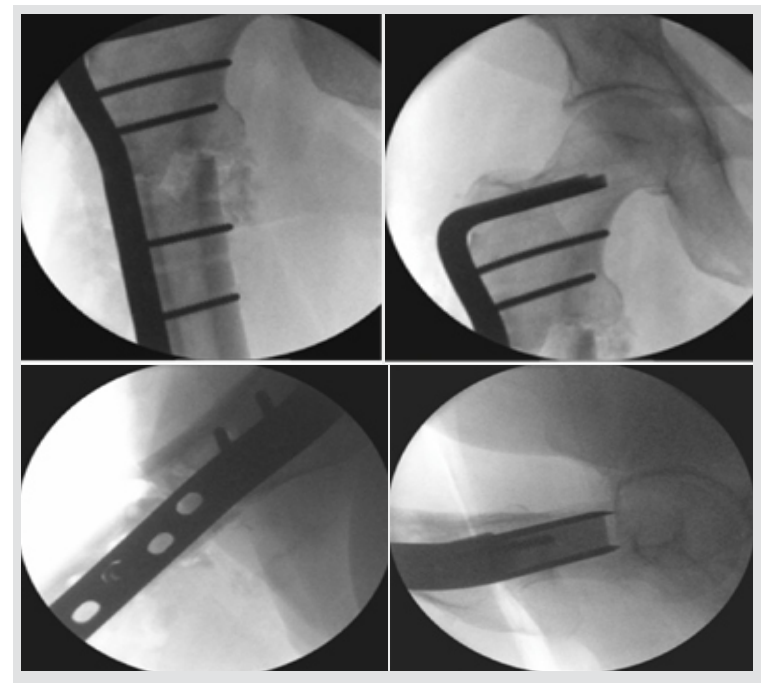

Figure 3: Intraoperative images showing initial revision to angled blade plate.
While abroad, the patient suffered another fall which completed the subtrochanteric fracture and was treated locally with a dynamic condylar screw (DCS) (Fig. 2). On his return to the UK at 2 weeks from the index procedure, it was apparent that the metalwork had failed, albeit with no clinical sign of infection. Revision fixation was undertaken 2 weeks later with the DCS being exchanged for a $95^{\circ}$ blade plate (Fig. 3). All microbiology cultures sent at the first revision surgery were negative, including for tuberculosis and fungi.

One month following revision, the patient presented with wound erythema and discharge. Radiographs were unremarkable but an ultrasound scan demonstrated a large collection along the length of the plate (Fig. 4). This was washed out and antibiotics were commenced, however, samples from this surgery grew no organisms. A second washout a month later for a similar presentation grew Staphylococcus epidermidis on enrichment culture only. Despite 3 months of antibiotics, the patient suffered ongoing issues with his wound and a third washout was performed which grew Staphylococcus haemolyticus. These samples were described as having an "unfavorable antibiogram" by microbiology colleagues. Of note, the radiographs now demonstrated failure of at least two of the diaphyseal screws of the blade plate.

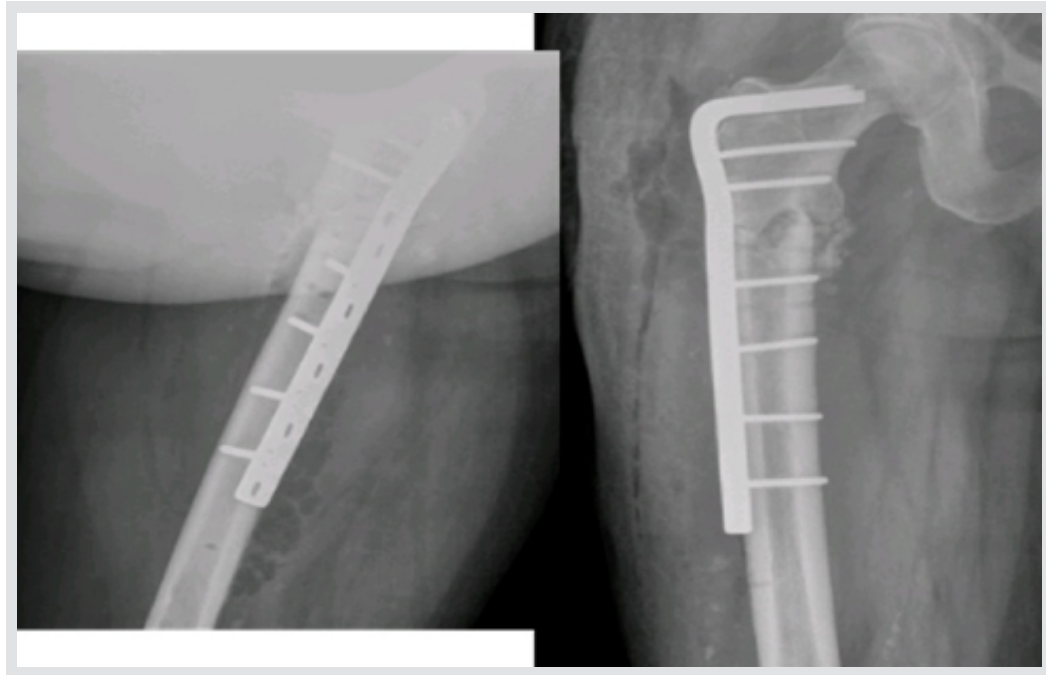

Figure 4: Coronal and oblique radiographs of the right proximal femur demonstrating a large soft-tissue collection. 


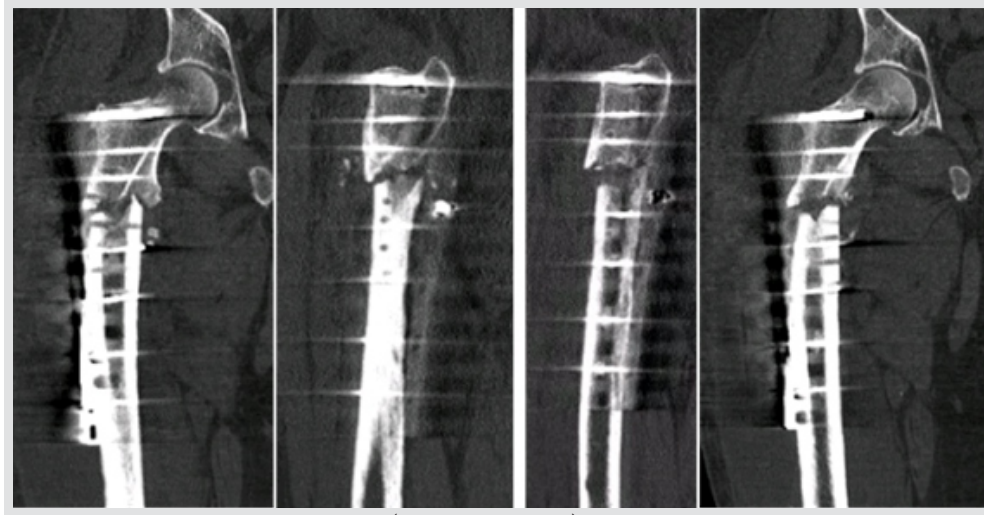

Figure 5: Computed tomography images (coronal and sagittal) confirming non-union and gas within the soft tissues.

A computed tomography scan performed 1 week later showed no evidence of bony union and gas within the soft tissues (Fig. 5). Revision was performed with resection of devitalized bone margins, compression, and stabilization with a full-length blade plate (Fig. 6). Unfortunately, 6 weeks after this second revision fixation, he developed tenderness and fullness again in his wound, and at this point, a wound swab grew Proteus mirabilis and Klebsiella pneumoniae. The patient was treated with IV piperacillin-tazobactam, vancomycin, and oral ciprofloxacin. He made steady progress, and in line with the patients' wishes, it was decided to attempt suppression until union.

Five months into antibiotic suppression, the patient suffered ongoing pain, wound erythema, and pyrexia. Plain radiography (Fig. 7) and cross-sectional imaging showed further gas within the soft tissues and non-union. Revision fixation was felt to be inappropriate due to resistant infection and the lack of union despite stable fixation. Taking into account, the patients' wishes to be pain free and to regain mobility, the decision was made to proceed with a two-stage proximal femoral replacement. All metalworks were removed, followed by proximal femurectomy and radical debridement with RIA treatment to the femoral intramedullary canal. The"'Cement-a-TAN" custom proximal femoral spacer was then made as described below and implanted (Fig. 8). All samples obtained at this time grew Proteus mirabilis, and the patient was commenced on a prolonged course of oral ciprofloxacin. Following discharge
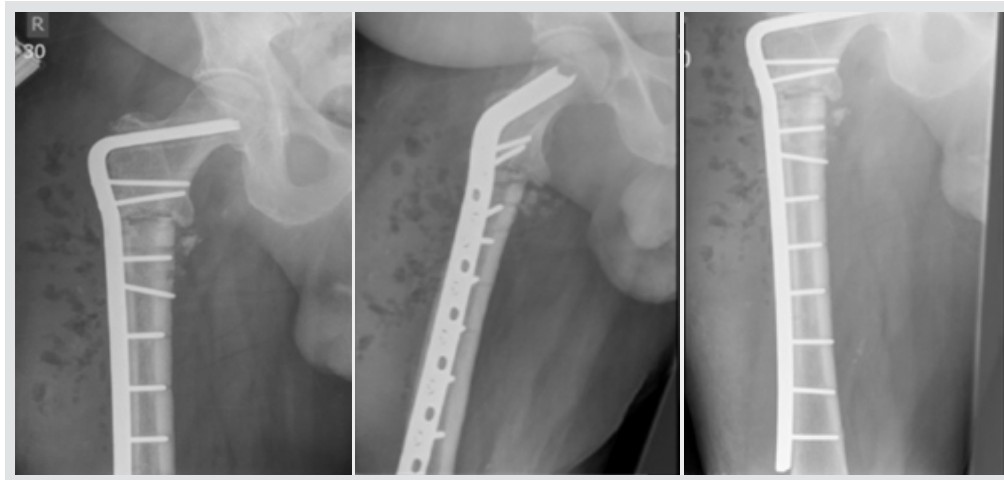

Figure 7: Coronal and oblique radiographs showing persistent gas within the soft tissues and non-union.

Journal of Orthopaedic Case Reports | Volume 11 | Issue 3 | March 2021 | Page 85-89
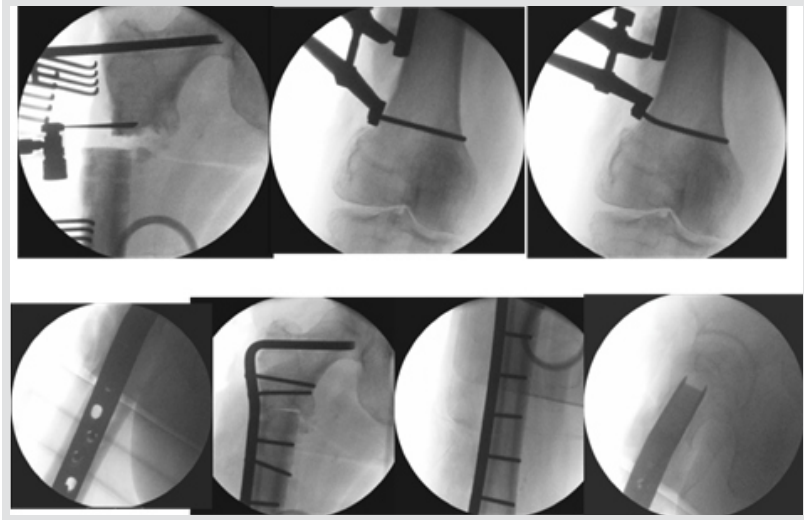

Figure 6: Intraoperative images showing resection of non-viable margins and revision fixation with angled blade plate. from hospital, the patient able to mobilize fully weight-bearing reported minimal pain and was able to return to driving. The surgical incision healed uneventfully.

The Cement-a-TAN remained in situ until the infection was felt to be controlled, with C-reactive protein persistently below $10 \mathrm{mg} / \mathrm{L}$. After 4 months, the Cement-a-TAN was explanted and converted to a proximal femoral replacement (Stanmore METS, Stanmore, UK) (Fig. 9). At 20 months follow-up, the patient was pain free and mobile, with a stable range of hip movement and a fully healed wound (Fig. 10).

\section{Cement-a-TAN construction technique}

A trochanteric entry cephalocondylar nail (Meta-TAN, Smith and Nephew, Memphis, USA) was templated using radiographs of the patients' contralateral side to ascertain leg length, offset, and femoral head size. The nail was pre-assembled ex vivo as per the standard surgical technique, with the integrated screws being inserted along with the set screw to lock the offset at the correct length. Antibiotic-eluting polymethyl-methacrylate (PMMA) bone cement was used to create a proximal femur-shaped temporary spacer, with culture-specific antibiotics being mixed into the cement. The head component was shaped using acetabular trials inside a glove to achieve a smooth finish (sized using radiographs of the contralateral femoral head and direct templating intraoperatively of the acetabulum), with manual

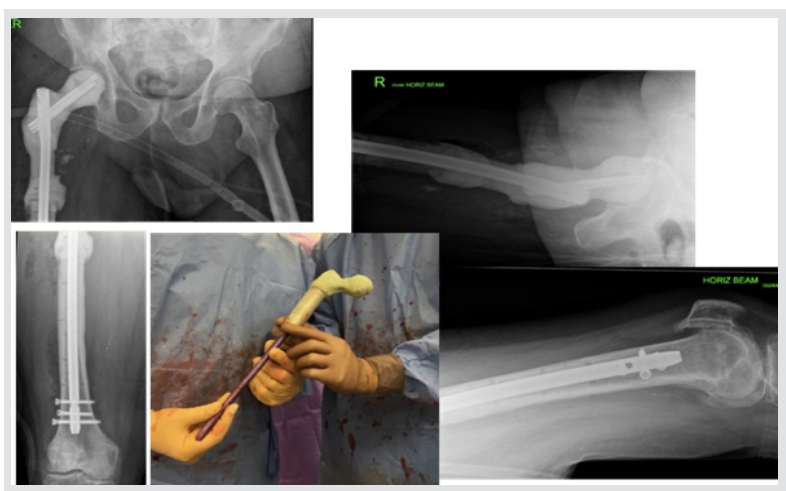

Figure 8: Construction and implantation of the "Cement-a-TAN." 


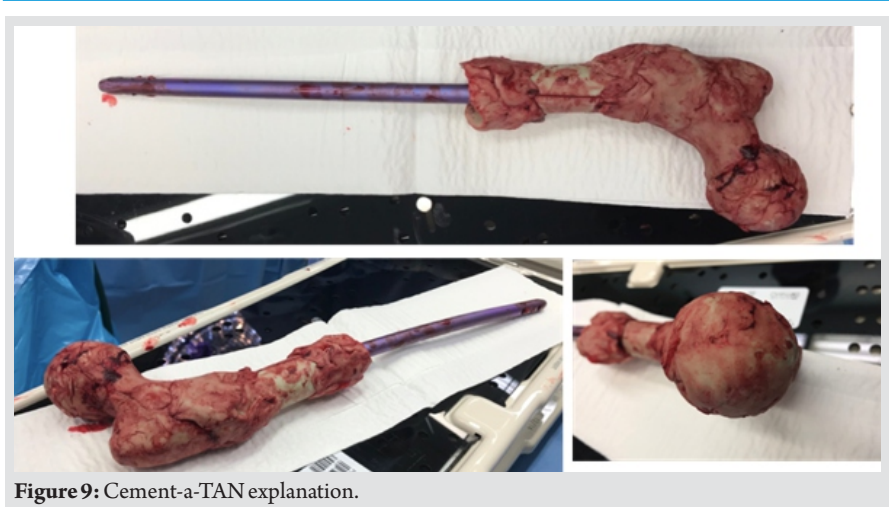

Figure 9: Cement-a-TAN explanation.

contouring of the trochanters. A $50 \mathrm{ml}$ bladder syringe was cut to the size of the resected diaphyseal portion, creating a cylinder that was placed around the nail, to replicate the diameter of the femur. The cylinder was filled with bone cement through side nozzle, with the plastic cut and removed once the bone cement cured. These three discrete parts were manufactured simultaneously to give a single composite construct around the pre-assembled nail. The residual femur was reamed using the Reamer Irrigator Aspirator (DePuy Synthes, Leeds, UK), and the construct inserted in the correct version and locked distally with polyaxial angle stable screws to provide both rotational and axial stability.

\section{Discussion}

Antibiotic-eluting temporary spacers are an important tool in the management of infected non-union of proximal femoral fractures. The goals of their use are delivery of high concentrations of antibiotics to the surrounding tissues, preservation of the softtissue architecture, reduce dead space, pain relief, and to allow a degree of weight-bearing with mobility while awaiting proximal femoral replacement $[3,4,6,8]$. We have demonstrated the "Cement-a-TAN" temporary spacer technique as an effective solution for challenging cases of significant proximal femoral bone loss in the context of infection.

There are few temporary spacing options described in the literature for such cases $[3,4,6,7]$. Antibiotic-eluting spacers can be either articulating or static, and preformed or custom made $[6,8]$. Static antibiotic spacers provide no formal connection between the femur and acetabulum, while articulating spacers allow motion to occur [8].Articulating spacers have the advantage of allowing weight-bearing which maintains a degree of function for patients and prevents disuse osteopenia amongst other complications of prolonged bed rest [6]. They also prevent significant limb shortening and contracture of the soft tissues between surgical stages [8]. As the interval between the first and second stages may be prolonged due to the requirement for extended antibiotic therapy, preservation of function is highly beneficial.

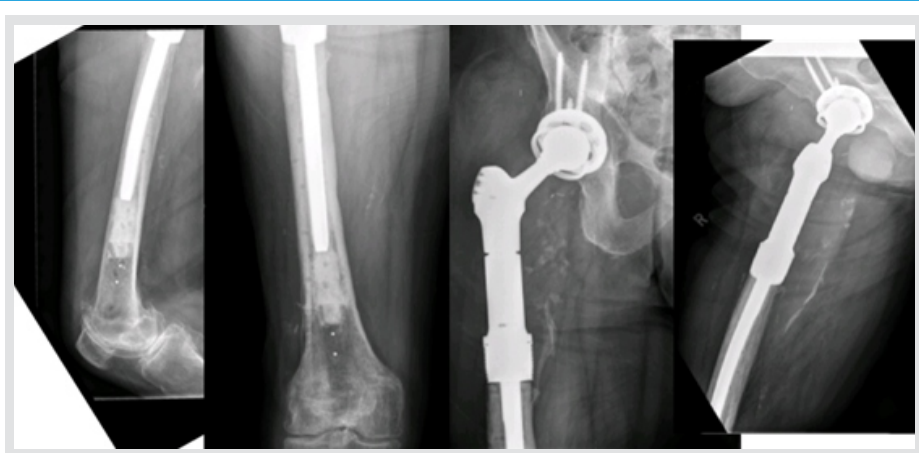

Figure 10: Coronal, lateral, and oblique radiographs showing latest follow-up at 20 months postprocedure.

Patient-specific articulating antibiotic spacers have advantages such as relative low cost, the ability to adjust length, offset, version, and femoral head diameter to match the anatomy of the patient but also to allow the surgeon to determine the type and dosage of antibiotic used [8]. Self-constructed articulating spacers can be made using a hip mold system such as the prosthesis with antibiotic-loaded acrylic cement (PROSTALAC) (Depuy Synthes, Leeds, UK), which uses a femoral stem core over which antibiotic cement is applied and subsequently clamped together by the mold $[9,10]$. The main drawback of hip mold systems is the limited choices of size $[4,8]$.

Custom spacers can also be crafted from bone cement impregnated with antibiotics formed around a metal endoskeleton $[5,6,8]$. If bone loss is minimal, standard spacers and commercially available devices (such as PROSTALAC) may be sufficient but in cases of extensive proximal femur bone loss, options become limited [3, $4,6]$. In this scenario, orthopedic surgeons have fashioned their own solutions. Rodriquez et al. and Kamath et al. took a short femoral intramedullary nail as a core and surrounded it with PMMA cement loaded with vancomycin and tobramycin $[3,4]$. Head size was estimated, and the cement was molded around the nail to approximate the shape of resected bone. In the case presented by Rodriguez et al., the nail was interlocked to provide rotational stability and the patient was able to transfer short distances until at 4 months she underwent reconstruction [3]. In 2017, Shields et al. combined an IMN and hand molded cement to create the femoral neck and head with a cement pseudoacetabulum. The patient was allowed to partially weight bear for 6 weeks [7].

Noh et al. in 2018 presented a similar case to our own, an infected non-union of a subtrochanteric fracture in a patient who had been taking bisphosphonates for 7 years. In this case, the authors planned to insert an antibiotic cement-coated nail as a singlestage procedure, but the patient's femoral canal was too narrow for a standard femoral nail so a tibial nail was used as an alternative. The patient was non-weight-bearing for 6 weeks postoperatively, progressing to partial weight-bearing when union was observed at 4 months. The patient was reported as pain free and ambulant with a frame at 4 years follow-up [5]. In 
2019, Mayes et al. adapted this idea by securing a modular body onto the cephalic portion of a long IMN using antibiotic laden PMMA [6]. The patient was then allowed to partial weight bear (up to 50\%) while waiting for the next stage. Mayes et al. argued that this method offers greater stability due to the combination of modular body and locked IMN One disadvantage of using a modular body is that it is only available in five sizes, which may not be suitable for all anatomical variants encountered.

The above custom-made articulating temporary spacers all preserve soft-tissue tension, elute antibiotics, and allow varying degrees of weight-bearing due to the use of an intramedullary nail which are designed to be load sharing. The Cement-a-TAN technique offers additional benefits. The use of polyaxial angle stable distal locking provides greater construct stability which, in turn, permits immediate full weight-bearing status and allows the patient to be effectively pain free while awaiting further surgery (the patient raised the possibility of keeping the Cement-a-TAN as a definitive solution!). The ability to lock the cephalic screws allows customized restoration of offset leading to enhanced stability. Furthermore, there is no need to order specialized equipment as the Cement-a-TAN can be made from material readily available in almost any orthopedic trauma unit.

\section{References}

1. Goff TA, Kanakaris NK. Management of infected non-union of the proximal femur: A combination of therapeutic techniques. Injury 2014;45:2101-5.

2. Cho JW, Kim J, Cho WT, Kent WT, Kim HJ, Oh JK. Antibiotic coated hinged threaded rods in the treatment of infected nonunions and intramedullary long bone infections. Injury 2018;49:1912-21.

3. Rodriguez H, Ziran BH. Temporary antibiotic cementcovered gamma nail spacer for an infected nonunion of the proximal femur. Clin Orthop Relat Res 2007;454:270-4.

4. Kamath AF, Anakwenze O, Lee GC, Nelson CL. Staged custom, intramedullary antibiotic spacers for severe segmental bone loss in infected total hip arthroplasty. Adv Orthop 2011;2011:398954.

5. Noh JH, Koh SJ, Lee KH. Treatment of proximal femur osteomyelitis occurred after proximal femoral nail antirotation fixation, with antibiotic cement-coated tibia intramedullary nail: A case report. Hip Pelvis 2018;30:4552.

Conflict of Interest: Nil

Source of Support: Nil

Consent: The authors confirm that informed consent was obtained

from the patient for publication of this case report
This means that it can be made with minimal planning and can be formed as a patient-specific device based on the relevant anatomy or bone resection. Minor complexities of the Cementa-TAN technique such as using the contralateral hip to template and the need for an experienced surgeon to perform this technique are offset by the multiple advantages outlined above. We believe that the combination of a cephalocondylar nail with culture-specific antibiotic-loaded cement is the most cost effective and beneficial option for these challenging cases.

\section{Conclusion}

The Cement-a-TAN is an excellent temporary spacing technique in staged proximal femoral replacement for infected non-union of the proximal femur where there has been significant boneloss.

\section{Clinical Message}

The Cement-a-TAN is an excellent, unique temporary spacing technique in staged proximal femoral replacement for infection in the proximal femur associated with significant bone loss. Orthopedic surgeons should be aware of how to construct this implant as a treatment option in these difficult cases.

6. Mayes W, Edwards PK, Mears SC. Management of severe proximal femur bone loss with a modular articulating antibiotic spacer. Geriatr Orthop Surg Rehabil 2019; 10:2151459319847399.

7. Shields D, Kong R, Gupta S, Mahendra A. A novel antibiotic spacer for significant proximal femoral loss surgical technique. Open OrthopJ 2017;11:508-16.

8. Sporer SM. Spacer design options and consideration for periprosthetic joint infection. J Arthroplasty 2020;35:S314.

9. Biring GS, Kostamo T, Garbuz DS, Masri BA, Duncan CP. Two-stage revision arthroplasty of the hip for infection using an interim articulated prostalac hip spacer: A 10 to 15-year follow-up study. J Bone Joint Surg Br 2009;91:1431-7.

10. DePuy Orthopaedics I. Prostalac Hip System; 2010. A v a i l a ble fro m : https://www.gsortho.org/system/files/resources/89/dp y_prostalac-hip_surgical_technique_0612-60-500r1.pdf.

\section{How to Cite this Article}

Scattergood SD, Fletcher JWA, Mehendale SA, Mitchell SR. Cement-aTAN Temporary Proximal Femoral Replacement for Staged Joint Replacement - A Case Report. Journal of Orthopaedic Case Reports 2021 March;11(3): 85-89. 